\title{
Estimation of Coherent Field Attenuation Through Dense Foliage Including Multiple Scattering
}

\author{
Il-Suek Koh, Feinian Wang, and Kamal Sarabandi, Fellow, IEEE
}

\begin{abstract}
Single-scattering theory is shown to be insufficient for the estimation of effective propagation constant in foliage at high microwave and millimeter-wave frequencies. Clusters of broad leaves and needles are treated as a unit scatterer whose ensemble forward scattering is used in Foldy's approximation to estimate attenuation rate in foliage. It is shown that single-scattering approximation overestimates forward scattering as high as 3-4 dB at 35 GHz.
\end{abstract}

Index Terms-Forest scattering, propagation constant, wave propagation.

\section{INTRODUCTION}

$\mathbf{I}$ $\mathrm{N}$ RADAR remote sensing of vegetation or estimation of wave propagation through forested environment an accurate electromagnetic scattering or wave propagation model is required. In such models, accurate estimation of attenuation rate is very important. From an electromagnetics point of view, a forest canopy can be considered as a random medium which consists of many dielectric objects such as branches, twigs, and leaves. These canopy constituents attenuate and scatter an incident electromagnetic field in a manner which depends upon the canopy particle size, density, locations, moisture content, the signal frequency and polarization. For most deciduous stands, broad leaves usually cluster rather densely at the end of branches, and are the dominant factors for attenuating microwave and millimeter-wave signals. Considering the much larger number of small needles on coniferous trees, it is clear that the needle clusters also contribute significantly to the attenuation. Other constituents such as branches, and trunks however, are distributed sparsely and therefore single-scattering theory is applicable for these scatterers.

Foldy's approximation has been widely used for the calculation of the mean-field in a random media like a forest because of its simplicity [1]. However, this formulation, which is based on single-scattering theory, is valid only for a sparse random media in which interactions among scatterers can be ignored. At high frequencies, where multiple scattering is a significant contribution, the accuracy of Foldy's approximation is questionable when it is applied to a dense medium like leaf-clusters. Various techniques that can be applied to dense media are summarized

Manuscript received October 19, 2002; revised April 8, 2003. This work was supported in part by the Defense Advance Research Projects Agency FCS Communications project under Subcontract S01-19 from the Pennsylvania State University and the Army Research Laboratory, and Collaborative Technology Alliance program under Contract DAAD19-01-02-0008.

The authors are with the Radiation Laboratory, Department of Electrical Engineering and Computer Science, The University of Michigan, Ann Arbor, MI 48109-2122 USA.

Digital Object Identifier 10.1109/TGRS.2003.813552 in [2]. In this letter, the accuracy of single-scattering theory for estimation of field attenuation is examined by applying a numerical technique, the method of moments (MoM). MoM is an exact solution (within numerical error) and is used to calculate the scattering from a cluster of leaves or needles. The result is then compared with a solution based on a coherent summation of the scattered fields from individual scatterers, which ignores multiple scattering. In order to calculate the ensemble average of forward scattering from clusters, exact numerical solution in conjunction with Monte Carlo simulation is used.

In examining the accuracy of single-scattering theory, approximate analytical formulations such as Rayleigh-Gans (lowfrequency solution for objects $\ll$ a wavelength), and volumetric physical optics approximation (VIPO) (high-frequency solution for objects $\gg$ a wavelength), for the calculation of scattering from a single leaf are also examined.

\section{APPROXIMATE SOlUtions FOR SCATTERING FROM A LEAF OR NEEDLE}

Broad leaves and needles are usually modeled by thin dielectric disks and thin elongated dielectric cylinders, respectively. However, exact electromagnetic scattering formulations do not exist for thin dielectric disks or thin finite dielectric cylinders. In practice approximate analytic solutions, based on high- and low-frequency techniques, have been used to calculate the scattered field from these kinds of shapes [3]. For scatterers with at least one dimension being much smaller than a wavelength, approximate formulations can generally be expressed in terms of a polarizability tensor [4]. The scattered field in the far-field region, for a long thin dielectric cylinder (needle), is given by

$$
\begin{aligned}
& \overrightarrow{\mathbf{E}}^{s} \sim-\frac{e^{i k_{0} r}}{r} \frac{\left(k_{0} a_{n}\right)^{2}}{4} l \\
& \quad \times \operatorname{sinc}\left[\frac{\left(\hat{k}_{i}-\hat{k}_{s}\right) \cdot \hat{l}}{2}\right]\left[\hat{k}_{s}\left(\hat{k}_{s} \cdot \vec{P}\right)-\vec{P}\right]
\end{aligned}
$$

where $l$ and $a_{n}$ are the length and the radius of the needle, respectively. Also for a thin elliptical disk (broad leaf) it can be shown that

$$
\begin{array}{r}
\overrightarrow{\mathbf{E}}^{s} \sim-\frac{e^{j k_{0} r}}{r} \frac{k_{0}^{2}}{4 \pi} \frac{2 t S_{d} J_{1}\left(\sqrt{\left(a_{l} A\right)^{2}+\left(b_{l} B\right)^{2}}\right)}{\sqrt{\left(a_{l} A\right)^{2}+\left(b_{l} B\right)^{2}}} \\
\times\left[\hat{k}_{s}\left(\hat{k}_{s} \cdot \vec{P}\right)-\vec{P}\right]
\end{array}
$$

where $t$ is the thickness of the elliptical disk; $a_{l}$ and $b_{l}$ are dimensions of the major and minor axes of the disk; and $S_{d}=\pi a_{l} b_{l}$ is its physical area. Also, $A=k_{0}\left(\hat{k}_{i}-\hat{k}_{s}\right) \cdot \hat{x}$ and $B=$ 
$k_{0}\left(\hat{k}_{i}-\hat{k}_{s}\right) \cdot \hat{y}$ where $\hat{k}_{i}$ is the propagation vector of an incident wave, and $\hat{k}_{s}$ is the unit vector specifying the direction of observation. $\vec{P}$ is a vector composed of the elements of the polarizability tensor, defined as $\left(\varepsilon_{r}-1\right)\left(P_{x x} E_{x}^{i} \hat{x}+P_{y y} E_{y}^{i} \hat{y}+P_{z z} E_{z}^{i} \hat{z}\right)$. For a thin circular cylinder and for a thin disk its diagonal elements are given respectively by

$$
\begin{aligned}
& P_{x x}=P_{y y}=\frac{2}{\varepsilon_{r}+1}, \quad P_{z z}=1 \quad \text { (needles) } \\
& P_{x x}=P_{y y}=1, \quad P_{z z}=1 / \varepsilon_{r} \quad \text { (leaves). }
\end{aligned}
$$

The above scattering formulations are obtained by extending the Rayleigh solution (low-frequency solution) to larger scatterers, by keeping one dimension much smaller than a wavelength. As will be shown, this solution (Rayleigh-Gans) for needles remains valid over a wide range of frequencies (if $a_{n} \ll \lambda$ ), however, a more accurate solution for broad leaves is needed. A solution for a broad leaf can be obtained by using polarization currents for an infinite dielectric slab having the same thickness, and dielectric constant of the disk. This solution will be referred to as volume integral physical optics (VIPO) solution. VIPO also predicts the same scattering formula as given by (2), however, the elements of $\vec{P}$ must be modified as follows:

$$
\begin{aligned}
& P_{x x}=P_{y y}=\frac{1+R_{h}}{1+R_{h} e^{i k_{z}^{\prime} t}} e^{i\left(k_{z}^{\prime}-k_{z}\right) t / 2} \\
& P_{z z}=\frac{1}{\varepsilon_{r}} \text { for h-pol. } \\
& P_{x x}=P_{y y}=\frac{1-R_{v}}{1-R_{v} e^{i k_{z}^{\prime} t}} e^{i\left(k_{z}^{\prime}-k_{z}\right) t / 2} \\
& P_{z z}=\frac{1}{\varepsilon_{r}} \frac{1+R_{v}}{1+R_{v} e^{i k_{z}^{\prime} t}} e^{i\left(k_{z}^{\prime}-k_{z}\right) t / 2} \quad \text { for v-pol. }
\end{aligned}
$$

where $k_{z}^{\prime 2}=k_{0}^{2}\left(\varepsilon_{r}-1\right)+k_{z}^{2}, k_{z}=\hat{k}_{i} \cdot \hat{z}$, and $R_{h}$ and $R_{v}$ are the Fresnel reflection coefficients for h-pol. and v-pol., respectively. If the thickness $t$ approaches zero, the VIPO reduces to the Rayleigh-Gans formulation (4). If the needle's radius is increased, the Rayleigh-Gans formulation (1) will no longer be valid, and numerical solutions must be used.

\section{Calculation of Attenuation Constant InCluding MULTIPLE SCATTERING}

At high frequencies when some or all dimensions of vegetation leaves become comparable to or larger than the wavelength, approximate analytical formulations may no longer be valid and multiple scattering among adjacent leaves becomes significant. For most deciduous trees, leaves in clusters are mostly parallel to each other but are somewhat random in their lateral positions. Pine needles on the other hand are more organized. A normal pine needle cluster has its needle buds distributed as three concentric spirals around a small stem and each have a tilt angle (angle from the stem axis), which decreases as the needle bud approaches the tip of the stem. Depending on the coniferous species the distance between each pair of needles can be as high as $5 \mathrm{~mm}$, larger than half a wavelength at $\mathrm{mm}$-wave frequencies, and the needle length can vary from $1-10 \mathrm{~cm}$. To examine the effect of multiple scattering on attenuation rate, MoM is applied to a cluster of broad-leaves and needles.

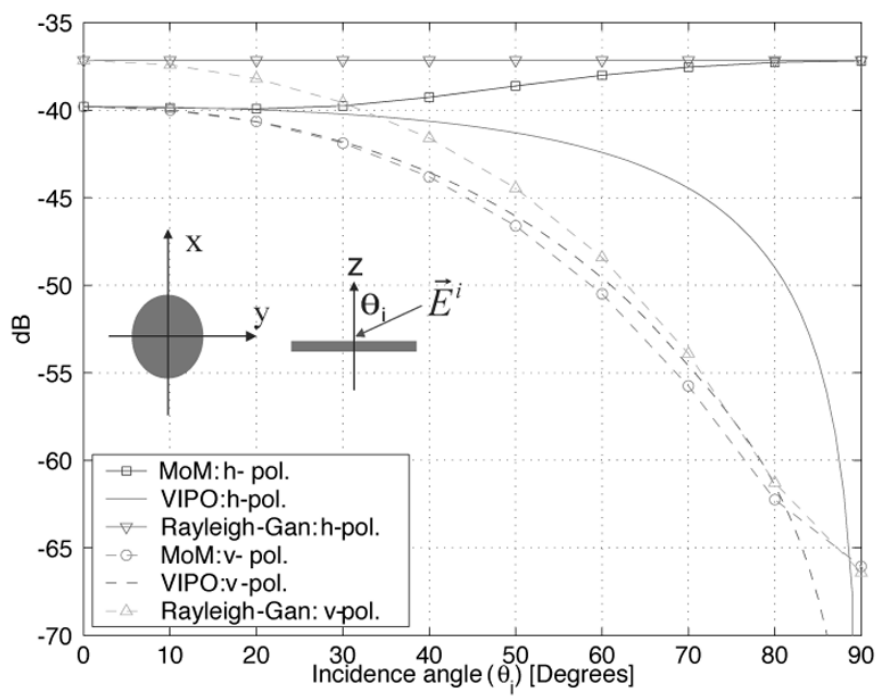

Fig. 1. Comparison of scattering matrix of circular dielectric disk in forward direction (magnitude) using Rayleigh-Gans, VIPO (2), and MoM for $S_{\mathrm{hh}}$, and $S_{\mathrm{vv}}$. The radius of the disk is $1.5 \mathrm{~cm}$, and its thickness is $0.02 \mathrm{~cm}$. Frequency is $10 \mathrm{GHz}$, and the dielectric constant of the disk is $26.6+i 11.54$.

The needle cluster stem is assumed to be oriented along the $z$ axis, in a local coordinate system. In a realistic model of a pine tree, the many needle clusters are oriented at different angles. However, the $3 N \times 3 N$ impedance matrix of each cluster, which is generated by discretizing a volumetric integral equation using a total of $N$ cells for needles of the cluster, is independent of the cluster orientation or the direction of the incident field for a given frequency. Therefore the impedance matrix needs only to be calculated and inverted once. The inverse matrix can be reused for various incident and observation directions. In the calculation of attenuation rate which is proportional to the average value of forward scattering, first scattering in the forward direction may be calculated for all incidence angles and then other cluster realizations can be generated for averaging. It should be noted that obtaining the inverse matrix, depending on the frequency, may require significant memory. To reduce the usage of memory the averaged inverse impedance matrix can be thresholded and stored. For example, for a needle cluster threshold with smaller than one percent $\left(10^{-2}\right)$ of the largest element of $Z^{-1}$, will produce $90 \%$ sparsity. The error associated with this thresholding procedure is less than $0.17 \mathrm{~dB}$.

\section{NUMERICAL RESULTS}

Foldy's approximation in the calculation of attenuation rate is based on a coherent average of forward scattering from individual scatterers (either single scatterers or clusters) and is given by $e^{i M_{m n} L}$, where $M_{m n}=(i 2 \pi N) /\left(k_{0}\right)\left\langle S_{m n}\right\rangle$, and $L$ is the slant distance through the vegetation layer. Also $N$ is the number of particles per unit volume; $S_{m n}$ is the forward scattering matrix element; and $m$ is the polarization of the scattered field component caused by an incident wave of polarization $n$ [1]. The accuracy of the method used for the calculation of scattering matrix in the forward direction determines the accuracy of the estimation of attenuation rate in the medium. Fig. 1 shows a comparison of the forward scattering matrix of a circular dielectric disk computed by Rayleigh-Gans, VIPO, and MoM. In 


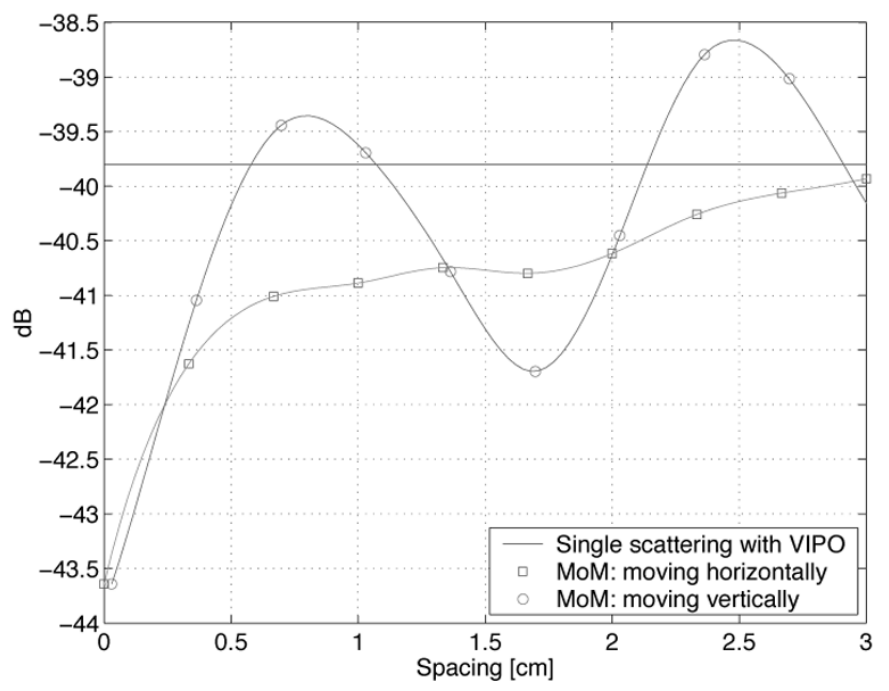

Fig. 2. Comparison of exact (MoM) results $\left(\left|S_{\mathrm{hh}}\right|^{2}\right)$ including multiple-scattering effect between two adjacent leaves with results from a single-scattering theory for two cases. In the case one, one leaf is moved moves horizontally, and in the other case one leaf moves vertically. Wave is normally incident.

Fig. 1, forward scattering $\left(S_{\mathrm{hh}}\right.$ and $\left.S_{\mathrm{vv}}\right)$ as a function of incidence angle $\left(\theta_{i}\right)$ is calculated for a thin circular disk whose radius and thickness are, respectively, 1.5 and $0.02 \mathrm{~cm}$ and which is oriented horizontally. Frequency is set at $10 \mathrm{GHz}$, and a dielectric constant of $26.6+i 11.56$ for the disk is chosen. As seen in Fig. 1, the Rayleigh-Gans solution overestimates forward scattering (attenuation rate) in this example. The VIPO solution is very accurate however, at near normal incidence angles, but its accuracy degenerates rapidly as the incidence angle approaches grazing. This is because VIPO does not account for diffraction from edges of the disk, and the VIPO currents approach zero at grazing incidence.

To examine the effect of multiple scattering for broad leaves, forward scattering from two adjacent identical circular leaves is calculated and shown in Fig. 2. Two cases are considered. In the first, the two leaves totally overlap, and then vertical separation is increased. In the second case, separation is fixed at $0.3 \mathrm{~mm}$, and one leaf is moved horizontally. It is shown that single-scattering theory overestimates the forward scattering by an amount as high as $3.8 \mathrm{~dB}$ for both cases. This observation is further confirmed by examining a cluster of leaves as well. For this simulation, ten leaves are randomly located inside a box whose dimensions are $10 \times 10 \times 10 \mathrm{~cm}$, and each leaf is assumed to be circular with a radius of $1.5 \mathrm{~cm}$. The unit normal of the dielectric disks is given a Gaussian distribution with zero mean and $15^{\circ}$ standard deviation with respect to the vertical axis. Monte Carlo simulation of forward scattering is shown to converge with only seven realizations. In Fig. 3, the results are shown which compare the full-wave MoM solution and its single scattering using MoM, Rayleigh-Gans, and VIPO. It is shown that single scattering using MoM, which calculates the forward scattering from an individual leaf, closely follows the results based on the full-wave MoM approach, but the Rayleigh-Gans and VIPO results are considerably different. Note that the full-wave MoM solution that includes multiple-scattering effects is always

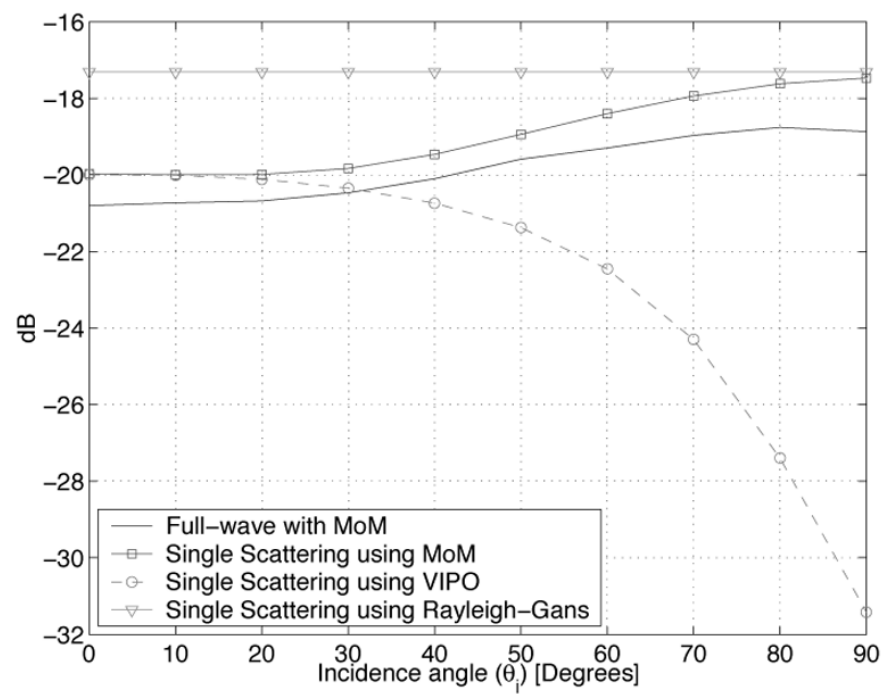

(a)

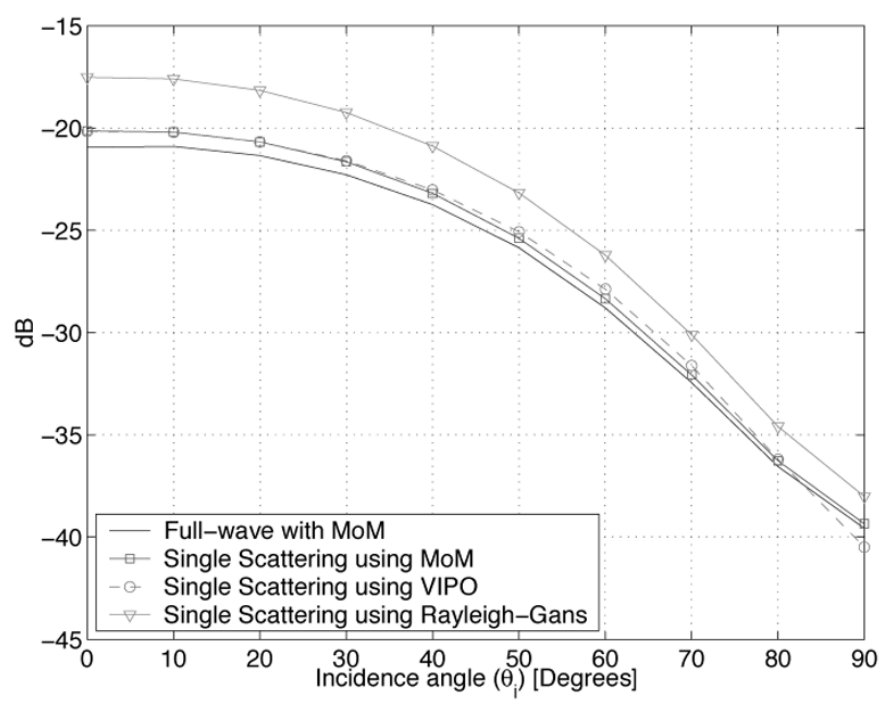

(b)

Fig. 3. Comparison of $S_{\mathrm{hh}}$, and $S_{\mathrm{vv}}$ that are calculated by four different methods. For this calculation, the effect of ten leaves is considered, and Monte Carlo simulation is carried out with seven realizations. Leaves are located inside a box whose dimension is $10 \times 10 \times 10 \mathrm{~cm}$. Density is $10^{4} / \mathrm{m}^{3}$. (a) $\left|S_{\mathrm{hh}}\right|^{2}$. (b) $\left|S_{\mathrm{vv}}\right|^{2}$.

lower than results predicted by any of the single-scattering results. For vertical polarization, single-scattering theory and the full-wave approach are not very different even at high incidence angles because the incident wave weakly couples to mostly horizontal leaves. The effect of multiple scattering (reduction in the attenuation rate as compared to the attenuation rate predicted by single-scattering theory) is enhanced as the size and number density of particles and/or frequency are increased.

Since the dimension of a needle is much smaller than a leaf, the effects of multiple scattering for a cluster of needles only become important at millimeter-wave frequencies. So for this case, a frequency of $35 \mathrm{GHz}$ is chosen. We consider a cluster of 12 needles each having a length $l=50 \mathrm{~mm}$, a transverse cross section radius $a_{n}=0.6 \mathrm{~mm}$, and a dielectric constant of $9+i 4$. Forward scattering is examined with and without the effect of multiple scattering. As before, single scattering 


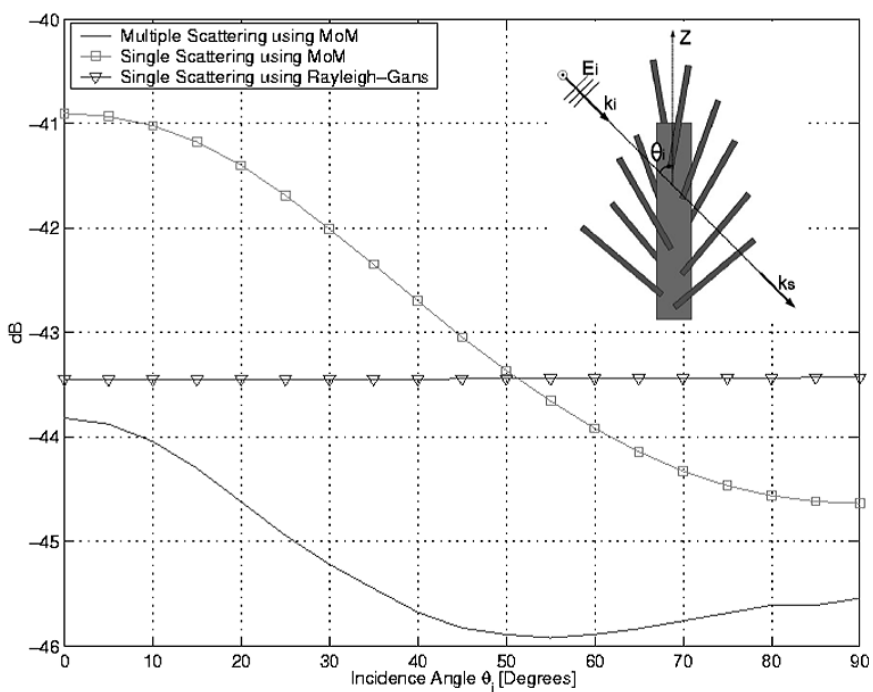

(a)

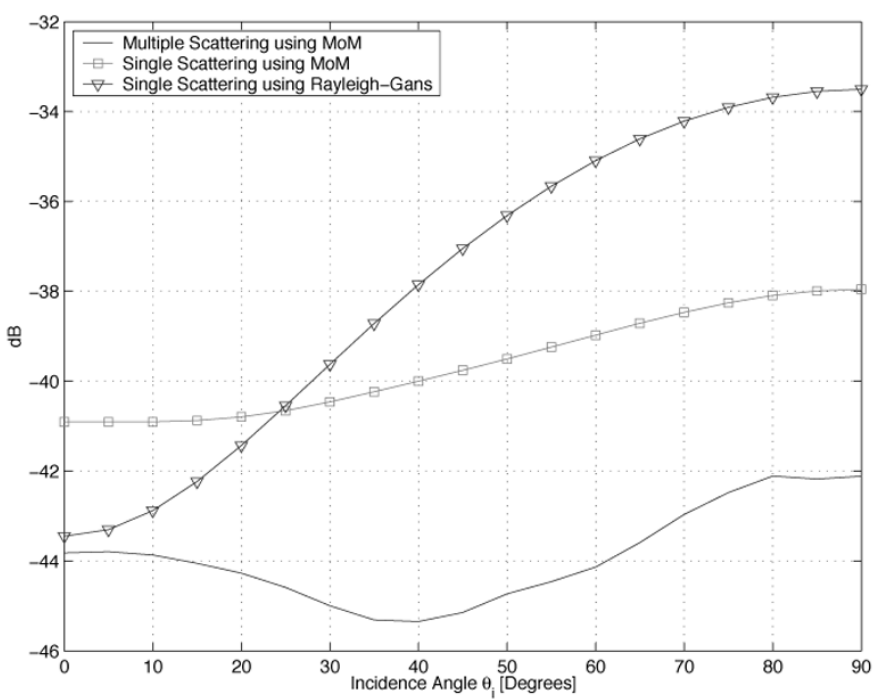

(b)

Fig. 4. Comparison of forward scattering $\left(S_{\mathrm{hh}}\right.$ and $\left.S_{\mathrm{vv}}\right)$ from a needle cluster as a function of incidence angles at $35 \mathrm{GHz}$. Nine iterations are realized. (a) $\left|S_{\mathrm{hh}}\right|^{2}$. (b) $\left|S_{\mathrm{vv}}\right|^{2}$. using MoM is computed by setting the off-diagonal block matrices in the impedance matrix to be zero. At these frequencies, needle parameters fall outside the Rayleigh-Gans region of validity $\left(2 k_{0} a_{n}\left|\sqrt{\epsilon_{r}-\cos ^{2} \theta_{i}}\right|<1[5]\right)$, and therefore even for a single needle, there may be some discrepancy between MoM and Rayleigh-Gans results. The effect of multiple scattering is expected to increase this discrepancy. Fig. 4 shows the scattering amplitude in the forward direction, $\left(\left\langle S_{\mathrm{hh}}\right\rangle\right.$ and $\left.\left\langle S_{\mathrm{vv}}\right\rangle\right)$. The incidence angle varies from $0^{\circ}$ to $90^{\circ}$ around the cluster. For this simulation, nine iterations are sufficient to achieve the mean value. At each realization, orientation angles of the needles are varied. As expected, the Rayleigh-Gans solution deviates from MoM by a maximum $2.5 \mathrm{~dB}$ for $S_{\mathrm{hh}}$ and $4 \mathrm{~dB}$ for $S_{\mathrm{vv}}$. In addition, it is shown that the multiple-scattering effect is significant, maximum $3 \mathrm{~dB}$ for $S_{\mathrm{hh}}$ and $5 \mathrm{~dB}$ for $S_{\mathrm{vv}}$.

\section{CONCLUSION}

The accuracy of single-scattering theory for estimation of attenuation rate of a dense medium like a leaf cluster is examined by comparing methods based on single-scattering theory and a full-wave solution MoM, which includes all multiple-scattering effects. Forward scattering simulations show that single-scattering theory usually overestimates the attenuation rate, and at high frequencies the discrepancy between the two methods increase. Monte Carlo simulations show that less than ten realizations are needed to compute the ensemble average for forward scattering. Inverse impedance matrices are thresholded and stored for the repeat computation of forward scattering at arbitrary incidence and cluster orientation angles.

\section{REFERENCES}

[1] L. Tsang, J. Kong, and R. T. Shin, Theory of Microwave Remote Sensing. New York: Wiley, 1985.

[2] P. R. Siqueira, "Wave propagation and scattering in dense random media," Ph.D. thesis, Univ. Michigan, Ann Arbor, MI, 1996.

[3] K. Sarabandi, "Electromagnetic scattering from vegetation canopies," Ph.D. thesis, Univ. Michigan, Ann Arbor, MI, 1989.

[4] R. F. Harrington, Time-Harmonic Electromagnetic Fields. New York: McGraw-Hill, 1961.

[5] Radar Cross Section Handbook, Plenum, New York, 1970. 